ment of pulmonary edema, but, as time went on they became unbalanced and weakened.

Based on the results of both clinical and experimental studies the pathogenesis of the clinical pulmonary edema occurring after a brain operation or a brain trauma was assumed to be as follows. The operation of trauma was directly responsible for the development of pulmonary hypertension, the prolongation of pulmonary circulation time, and the increase in the permeability of the pulmonary capillaries. At the same time, the operation or the trauma directly caused the changes in renal or hepatic circulation and endocrine regulation, inducing anuria, disturbance of water and electrolytes balances. The combination of these factors accelerated a selective pulmonary edema of an acute type.

Pulmonary edema occurring after an operation of cardiopulmonary diseases was often caused by a misuse of blood transfusion during and after operation. At this time something different was assumed as to the mechanism in development of the pulmonary edema. The continual rise of pulmonary arterial pressure and the remarkable lowering of colloidal osmotic pressure of plasma are the chief factors causing pulmonary edema. Once the pulmonary edema was induced, it became a stress, resulting indirectly in renal circulatory failure, discrepancy of endocrine organs, increase of the permeability of pulmonary capillaries and the further promotion of the pulmonary edema.

Pulmonary edema occurring after an operation of abdominal diseases with metabolic disorder was characteristically caused by a tremendous increase of permeability of the pulmonary capillaries.

\title{
The Mechanism of the Development of Acute Pulmonary Edema in Lung Surgery
}

\author{
Y. Sagawa and C. Nagaishi \\ Department of Surgery and Tuberculosis Research Institute \\ Kyoto University, Kyoto, Japan
}

According to clinical observation, pathogenesis of acute postoperative pulmonary edema in lung surgery can be roughly classified into two categories. One is ventilatory insufficiency and the other is severe hemorrhage, excessive transfusion and infusion.

We gave these causative factors to dogs as experimental loads, and observed the mechanism of transudation from pulmonary blood vessels. The results were presented at the 3rd Meeting of Asian-Pacific Congress of Cardiology. Since acute pulmonary edema is a result of unbalance between transudation of blood component and absorption of the transudate, it is necessary for the study of acute pulmonary edema to demonstrate how the absorption of transudate occurs when experimental animals are exposed to these causative factors of acute pulmonary edema.

Since there is no other practical way to demonstrate directly where and how blood component enters into pulmonary blood vessels from surrounding lung interstitium, we studied the absorptive capacity of pulmonary blood and lymph vessels for transudate by observing how saline solution added with $\mathrm{P}^{32}$ as a tracer and instilled into alveoli can be transported from outside to inside of vessels. One or 
$2 \mathrm{ml}$. of physiologic saline solution containing 0.4-0.6 mc. $\mathrm{P}^{32}$ was instilled as rapid as possible through a small canule inserted down the right main bronchus of a dog under various experimental conditions. In some animals only blood samples were obtained through femoral artery, whereas both blood and lymph samples were drawn simultaneously from femoral artery and right lymphatic trunk in other animals. Absorptive capacity of pulmonary blood vessel or lymph vessel was obtained by measuring specific activity of blood or lymph samples.

We chose the ventilatory insufficiency as experimental conditions or loads out of the various pathogenetic factors of acute pulmonary edema above stated. They consisted of hypoxia, hypercapnea, hypoxia combined with hypercapnea, hypoxia with open thorax and increased airway resistance.

Both experimental groups on hypoxia alone with inhalation of about $10 \% \mathrm{O}_{2}$ and on hypercapnea alone showed a decreased absorptive capacity which turned back to control level on discontinuing the loads. Both groups on hypoxia $\left(10 \% \mathrm{O}_{2}\right)$ combined with hypercapnea and on hypoxia combined with open thorax showed marked decrease in absorptive capacity which failed to turn back on discontinuing the load in some animals.

The absorptive process through Jymphatic system was so slow and limited that the amount was negligible compared with that of blood vessels. There is an intimate relation between absorption and transudation in and out of pulmonary vascular bed and it is conceivable that the decrease in absorption indicates the increased transudation. Therefore, it can be stated that the absorptive function of pulmonary blood and lymph vessel has only minor significance for the development of acute pulmonary edema.

\title{
Pulmonary Edema as a Cause of Surfactant Deficiency
}

\author{
S. I. Said, C. M. Banerjee, W. R. Harlan, Jr., and M. E. Avery \\ Department of Medicine, Medical College of Virginia, \\ Richmond, Virginia, U.S.A.
}

The induction of pulmonary edema in anesthetized dogs led to a patchy impairment of pulmonary surface activity, as measured by altered force-area relations of saline extracts. Surface activity was decreased whether pulmonary edema was induced by rapid infusion of dextran, or by injection of alloxan or ANTU. The altered surface properties were of sufficient magnitude and extent to result in abnormal pressure-volume relations of degased lobes. Morphologically, areas of decreased surface activity were usually hemorrhagic but not foamy and had smaller or occluded alveoli.

Loss of surface-active lining in foam was an important factor in causing the deficiency of surface activity with pulmonary edema. This was shown by a less marked impairment of surface activity when foaming was prevented (by degassing a lobe before, and occluding its airway during induction of edema). The persistence of some degree of surface tension alteration in the absence of foaming suggested that the mere transudation of plasma into the alveolar spaces somehow interfered with surfactant. 